\title{
EVALUACIÓN PILOTO DE TOXICIDAD AGUDA DEL RIO FUCHA
}

\author{
PILOT EVALUATION OF ACUTE TOXICITY OF FUCHA RIVER
}

\section{Didiana Vanessa Giraldo Osorio}

Est. Ingeniería Ambiental, Semillero Metamorfo, Universidad Nacional Abierta y a Distancia dvgiraldoo@unadvirtual.edu.co

\section{Juan S. Chiriví-Salomón}

Docente ocasional de la Cadena de Formación Ambiental, Grupo de Conservación, Bioprospección y Desarrollo Sostenible, Universidad Nacional Abierta y a Distancia juan.chirivi@unad.edu.co

\section{Resumen}

El Río Fucha es una corriente que atraviesa la ciudad de Bogotá y que con el tiempo se ha vuelto receptora de distintos tipos de vertimientos y desperdicios. A su vez, implementar acciones de recuperación y conservación sobre el mismo implica una disminución progresiva de la afectación dada al paisaje urbano y del Río Bogotá donde desemboca. Es por lo anterior que, este estudio tuvo la intención de realizar una evaluación piloto de la toxicología aguda de sus aguas a través de un bioensayo utilizando semillas de Lactuca sativa L., que es un habitual modelo vegetal para evaluar fitotoxicidad. El bioensayo consistió en la exposición de semillas certificadas a una muestra de agua del río, en diferentes concentraciones y utilizando dos controles de agua salina y agua potable. Se observó una evidente inhibición de la germinación, llegando a un máximo de $30 \%$ y que tiende a disminuir a medida que disminuye la concentración de la muestra colectada. También se observó una afectación en la longitud radicular dada por una inhibición radicular del $52,4 \%$ en la concentración más alta de la muestra. Morfológicamente, se encontró evidencia de la toxicidad sobre las semillas al observar ápices radiculares con necrosis moderada, poco desarrollo en los pelos absorbentes de la raíz y necrosis en al menos

DOI: https://doi.org/10.22490/26653176.2782 


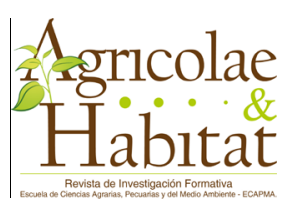

uno de los cotiledones. Este estudio es una línea base toxicológica para desarrollar proyectos de semillero o como opción de grado, e implementar a mediano plazo programas de saneamiento para recuperar el río Fucha. Se recomienda que estos productos sean vinculados a estudios composicionales que permitan relacionar de manera directa los hallazgos toxicológicos con agentes contaminantes específicos.

Palabras Clave: Bioensayo; Calidad De Agua; Lactuca sativa L.; Toxicología.
Abstract
The Fucha River is a water torrent that flows westward through the city into the Bogotá River. A huge quantity of landfills and wastes have been throwing over time, which creates a recalcitrant environmental affectation in this river. Remediation and conservation strategies are needed to solve this problem, which in turn mitigate the progressive affectation in the urban landscape and Bogotá River. That's why we had the intention to make a pilot evaluation of acute toxicology of a sampled water from the river through an exposal bioassay on Lactuca sativa $L$. seeds. This consisted in the exposal of seeds to the sampled water at different concentrations. Two controls with a salt water and drinking water were performed to guarantee the truthfulness of obtained data. An inhibition on the germination resulted evident with a $30 \%$ of maximum inhibition at the highest tested concentration. This inhibition tended to decreased over tested concentration. We also found an affectation in the root length due to a radicular inhibition of $52,4 \%$ in the highest tested concentration. In a morphological level, a direct toxic affectation was observed on roots and cotyledons. Moderated necrosis on radicular apex, low development on absorbent hairs, and necrotic tissue in at least one cotyledon, were the main observed morphological affectation. This study remarks a toxicological baseline of research for the formulation and development of seeds projects or thesis about the contamination of Fucha River. Chemical compositional studies are needed to link our results with specific pollutants.

Keywords: Bioassay; Lactuca sativa L.; Toxicology; Water Quality. 


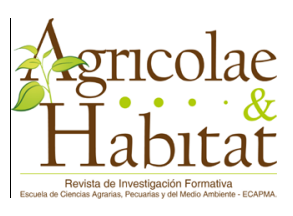

\section{Introducción}

A pesar de los convenios y normas implementados para la reducción de residuos peligrosos en Colombia, su inadecuado manejo sigue siendo un tema persistente debido al volumen poblacional involucrado (Tovar Artunduaga et al., 2015). Entre los residuos industriales de mayor importancia en Colombia, se encuentran aquellos ricos en metales pesados, compuestos fenólicos, hidrocarburos, entre otros compuestos recalcitrantes, que son generados principalmente de los sectores minero, energético y agrícola (Reyes et al., 2016; Beltrán Pineda y Gómez Rodríguez, 2014). En Bogotá, esta situación ha tenido relevancia por la cantidad de vertimientos que son depositados en el río Fucha, especialmente a la altura de la localidad de Puente Aranda, donde se ha reportado un alto índice de contaminación por metales pesados (Alcaldía local Puente Aranda, 2012; Pachón y Sarmiento Vela, 2008). Así, desde el 2002 se viene estudiando los resultados del seguimiento de la calidad del agua a través de la Red de Calidad Hídrica, instrumento para la aplicación de acciones de saneamiento, implementación de tecnología para mitigar la contaminación hídrica y ambiental, la gestión efectiva y el involucramiento de actores para su mejoramiento (Moreno Rojas, et al., 2008). Las acciones implementadas a nivel municipal por el Secretaría Distrital de Planeación, se encaminan sólo a la recuperación de espacios viales, como lo ha sido la ciclo-ruta paralela al río Fucha (SDP, 2009). Por lo anterior, este estudio buscó evaluar de forma rústica (en condiciones ambientales no controladas) la toxicología aguda de las aguas del Río Fucha a través de bioensayos con semillas de lechuga (Lactuca sativa L.). Este trabajo se realizó en el curso de Toxicología Ambiental del periodo 8-3 del 2018, bajo el soporte académico de la Escuela de Ciencias Agrícolas, Pecuarias y del Medio 


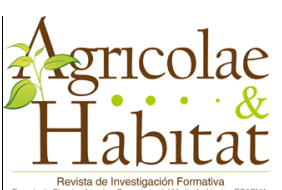

Ambiente, y con la intención de generar material que sirva de base para un futuro trabajo de grado del programa de Ingeniería Ambiental.

\section{Metodología}

\section{Lugar y condiciones de estudio}

El estudio realizado es de tipo descriptivo al analizar el potencial estado de contaminación del río Fucha a través de un método toxicológico de bioensayo en condiciones ambientalmente no controladas. Se estableció como punto de muestreo en el río Fucha a la altura de la Localidad de Puente Aranda, específicamente el tramo del sector relacionado con el Parque Milenta, delimitado por la Calle $8^{a}$ sur, Calle 14 sur y Diagonal 2 sur $\left(4^{\circ} 37^{\prime} 03,8^{\prime \prime} \mathrm{N} 74^{\circ} 07^{\prime} 28,7^{\prime \prime} \mathrm{W}\right.$ (Google, s.f.) (Figura 1). La temperatura ambiente promedio de este lugar es de $20{ }^{\circ} \mathrm{C}$ (AccuWeather, 2018).

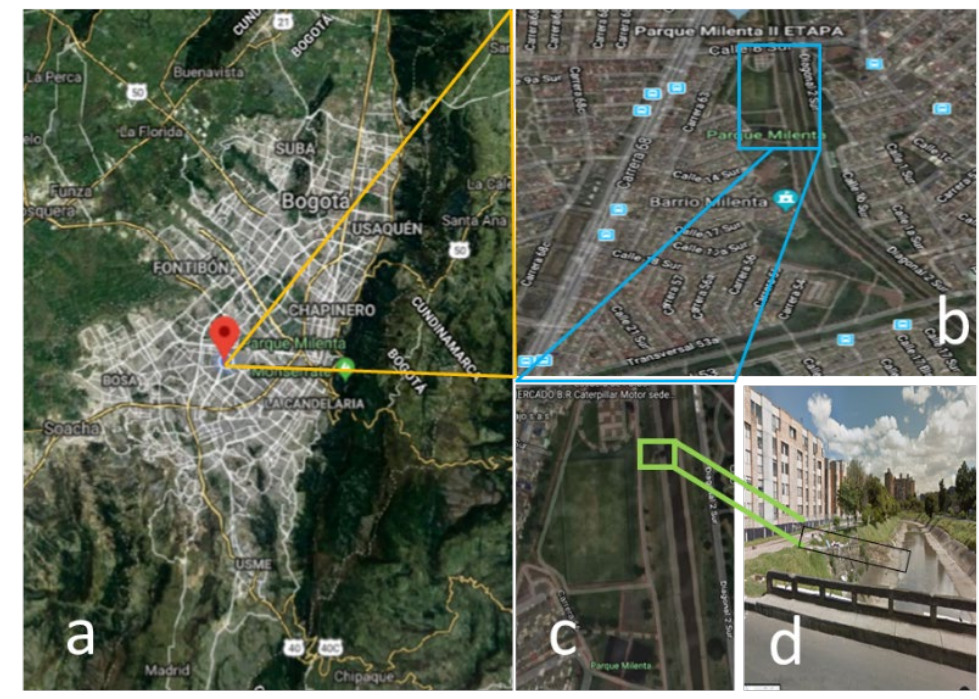

Figura 1. Lugar de muestreo: a) Ubicación del río Fucha en Bogotá. b) Ubicación de zona de muestreo en el parque Milenta c) Entrada a la zona de muestreo, en el parque Milenta, d) Fotografía de la entrada a la zona de muestreo (Google, s.f.). 


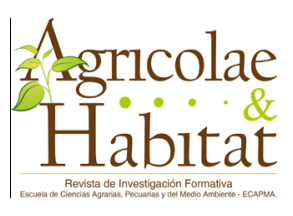

\section{Muestreo}

Se realizó un único muestreo, recolectando $1 \mathrm{~L}$ de agua del río con un balde. La muestra fue envasada y rotulada en un recipiente plástico, previamente lavado con abundante agua limpia. Se transportó la muestra en condiciones de aislamiento térmico, utilizando una nevera de poliestireno expandido, hasta el lugar de experimentación. El experimento se realizó en un lugar residencial, con condiciones básicas de protección personal. La colecta de datos se realizó conforme al protocolo establecido por el Instituto de hidrología, meteorología y estudios ambientales (IDEAM, 2002).

\section{Material biológico y verificación de viabilidad}

Las semillas de Lactuca sativa $L$. fueron adquiridas a través del proveedor C.I. Futura Internacional S.A.S., que las certifican con un porcentaje de germinación (\% G) de $85 \%$, una pureza de $99 \%$ libre de fungicidas o plaguicidas y con fecha de expedición de diciembre de 2017. Para verificar estas condiciones, se probó la viabilidad de las semillas haciendo un montaje con 3 réplicas con agua limpia, de las cuales se debía obtener un \% G superior al $85 \%$ para iniciar los montajes. Se determinó el porcentaje de inhibición de germinación (\% IG) como lo describen Sobrero y Ronco (2004).

\section{Bioensayo de toxicidad aguda}

Se procedió a la realización del bioensayo, y sus respectivos cálculos, como lo describe Sobrero y Ronco (2004), el cual consistió en la realización de un montaje casero utilizando platos desechables rotulados, papel de cocina y bolsas plásticas (Figura 2). Estos elementos sirvieron de soporte para las semillas, brindar condiciones de humedad y protección de la luz solar, respectivamente. Se realizaron dos controles, uno negativo ( $\mathrm{C}-$ ) con agua potable y otro positivo $(\mathrm{C}+)$ neutralizado con $\mathrm{NaCl}$. Cinco diluciones de la muestra con concentraciones al $100 \%$, $30 \%, 10 \%, 3 \%$ y $1 \%$ fueron utilizados para evaluar la toxicología de la muestra colectada. En total se realizaron 7 tipos de muestras con 2 réplicas para un total de 21 montajes. La incubación se realizó a temperatura ambiente y durante 5 días. Se tomaron medidas radiculares y del hipocólito para estimar los porcentajes de inhibición radicular (\% IR) y de inhibición del hipocólito (\% IH). Para validar las poblaciones de datos, se realizó un análisis de varianza de un solo factor con una significancia del $5 \%$ y una prueba de Tukey, para cada una de 


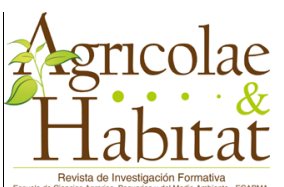

las medidas realizadas y procesando los datos con la herramienta de Análisis de Excel Microsoft (c) 2016.

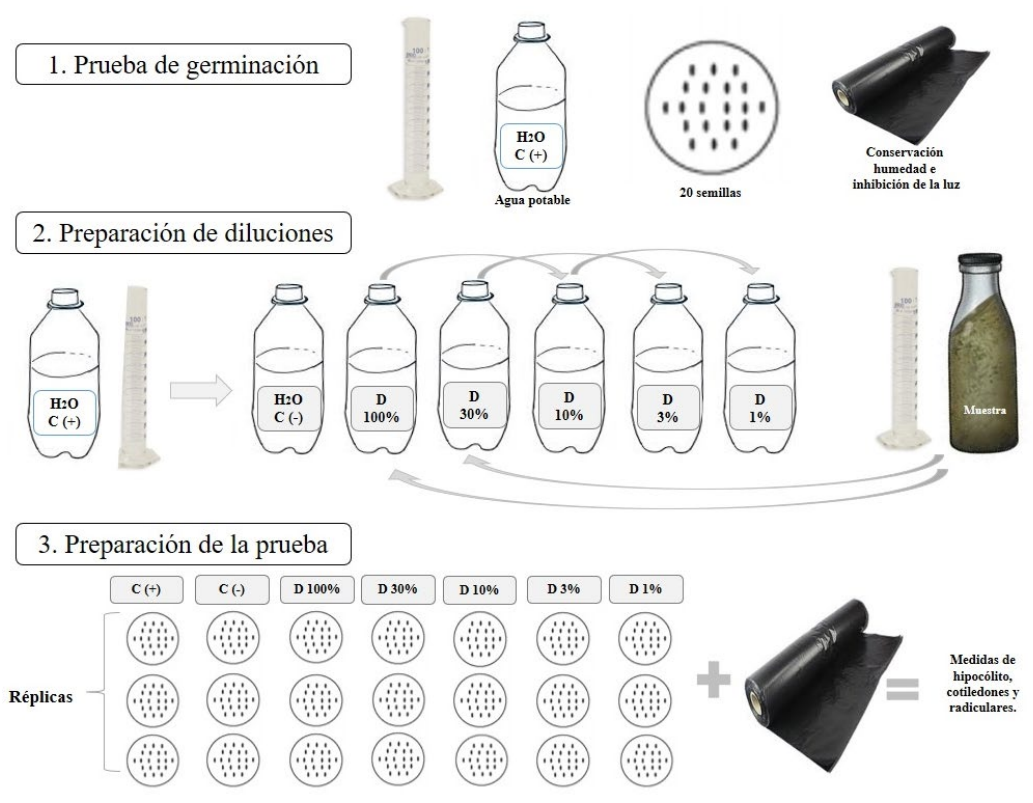

Figura 2. Ilustración de montaje de bioensayo utilizando semillas de Lactuca sativa L.

\section{Discusiones y resultados}

Durante el muestreo, se observó un alto grado de deterioro en la zona debido a actividades posiblemente ilegales de lavadero de vehículos, inadecuado manejo de residuos sólidos, acumulación de residuos y asentamiento de habitantes de la calle (Figura 3). 

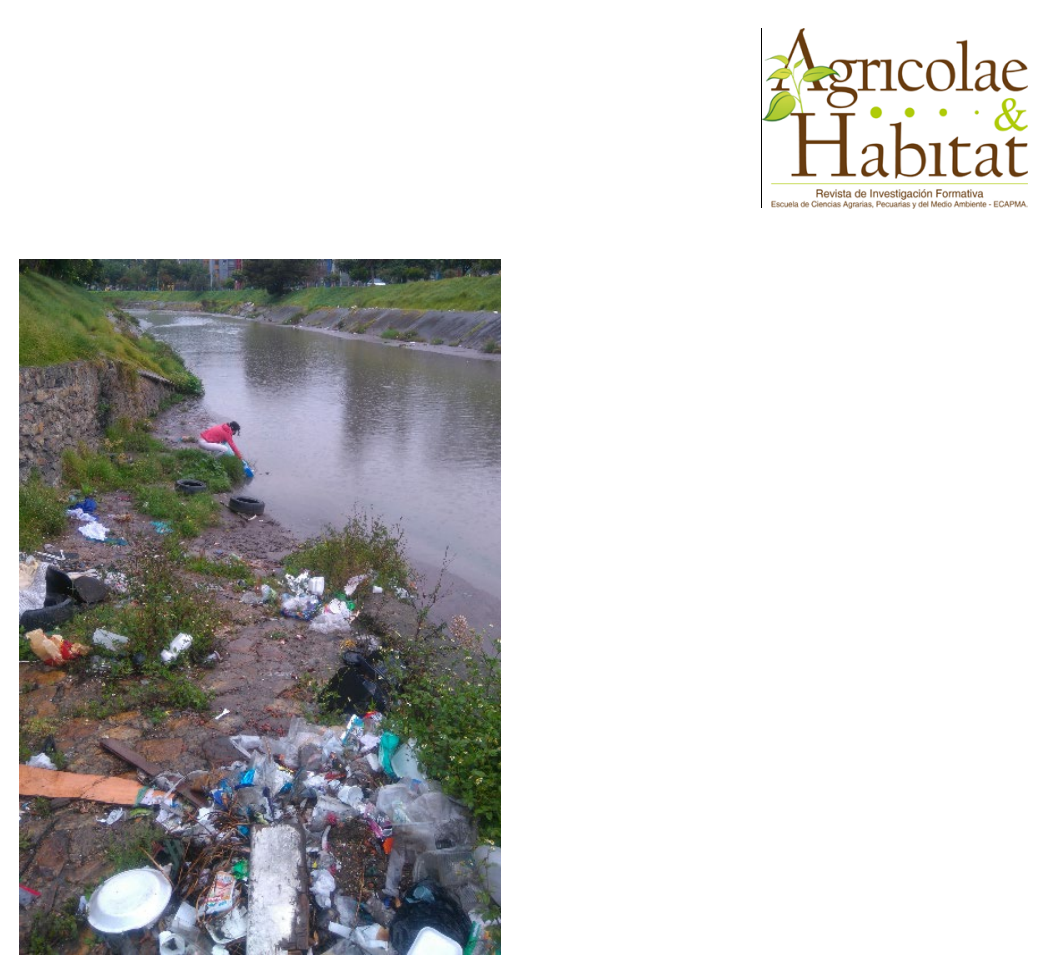

Figura 3. Fotografía de punto de muestreo.

Los datos de número de semillas germinadas mostraron diferencias estadísticas entre los tratamientos y los controles (valor $p<0.05$ ). Por otro lado, los datos de longitud radicular resultaron diferentes entre los controles y los tratamientos de máxima y mínima concentración (valor $p$ $<0.05$ ), pero no con diluciones intermedias (valor $\mathrm{p}=0.77$ ). Finalmente, los datos de longitud del hipocólito no fueron estadísticamente diferentes entre ninguno de los tratamientos (valor $\mathrm{p}=$ 0.16 ) dada la alta varianza de algunos tratamientos. Los resultados de las pruebas se registran en la Tabla 1.

Tabla 1. Resultados obtenidos en las pruebas de toxicidad realizadas con Lactuca sativa $\mathrm{L}$.

\begin{tabular}{|c|c|c|c|c|c|}
\hline \multicolumn{4}{|c|}{ CÁLCULOS PRUEBA DE TOXICIDAD } & Letal & Subletal \\
\hline Ensayo & $\% \mathrm{G}$ & Raíz (cm) & Hipocótilo $(\mathrm{cm})$ & $\%$ IG & $\%$ IR \\
\hline$C(+)$ & $\begin{array}{c}93,3( \pm \\
0,6)\end{array}$ & $2,6( \pm 0,5)^{\mathrm{a}}$ & $5,3( \pm 4,0)^{a, b}$ & & \\
\hline$C(-)$ & $\begin{array}{c}83,3( \pm \\
0,6)^{\mathrm{a}}\end{array}$ & $2,1( \pm 0,3)$ & $1,5( \pm 0,5)^{\mathrm{a}}$ & & \\
\hline
\end{tabular}




\begin{tabular}{|c|c|c|c|c|c|c|}
\hline D100\% & $\begin{array}{c}58,3( \pm \\
0,6)^{\mathrm{a}}\end{array}$ & $1,0( \pm 0,1)^{a, b}$ & $3,9( \pm 1,1)$ & 30,0 & 52,4 & $-152,2$ \\
\hline D30\% & $\begin{array}{c}63,3( \pm \\
1,5)\end{array}$ & $2,8( \pm 0,1)^{b}$ & $2,4( \pm 0,6)^{b}$ & 24 & $-33,3$ & $-58,7$ \\
\hline D10\% & $\begin{array}{c}63,3( \pm \\
2,5)\end{array}$ & $2,6( \pm 0,4)^{\mathrm{b}}$ & $1,6( \pm 1,0)^{b}$ & 24 & $-25,4$ & $-4,3$ \\
\hline D3\% & $\begin{array}{c}70,0( \pm \\
1,0)\end{array}$ & $2,6( \pm 0,3)^{b}$ & $2,8( \pm 0,2)$ & 16 & $-25,4$ & $-82,6$ \\
\hline D1\% & $\begin{array}{c}80,0( \pm \\
1,0)\end{array}$ & $3,4( \pm 0,5)^{\mathrm{b}}$ & $3,0( \pm 0,2)$ & 4 & $-61,9$ & $-97,8$ \\
\hline
\end{tabular}

\% IG: Inhibición de germinación, \% IR: Inhibición radicular, $\% \mathrm{IH}$ : Inhibición del hipocolito

Los superíndices de la misma letra indican las diferencias significativas entre los tratamientos de los datos obtenidos por la prueba de Tukey.

Como se puede evidenciar, la prueba de germinación resultó en un 93,3 $\%$, porcentaje efectivo según Sobrero y Ronco (2004) que establece que el porcentaje mínimo necesario para la verificación de la semilla es de $85 \%$. Por otro lado, se observó una clara tendencia tóxica letal al alcanzar una inhibición de la germinación del 52,4 \%, que disminuye a medida que se diluye la muestra de agua (Figura 4A). En relación a los datos obtenidos de la raíz, y aunque estadísticamente no se establecieron diferencias significativas entre los tratamientos D30\%, $\mathrm{D} 10 \%$ y $\mathrm{D} 3 \%$ (valor $\mathrm{p}=0.71$ ), si se identificó una diferencia con las dosis extremas y se observó un crecimiento anormal en toda la población (ápices radiculares con necrosis moderada, pelos absorbentes de la raíz, necrosis en al menos uno de los cotiledones); estudios sobre la toxicidad de varias semillas demuestran efectos de exaltación u hormesis, que explica las notorias diferencias en D100\% y D1\%, pero no son contundentes en efectos significativos de inhibición gradual (Bohórquez Echeverry y Campos Pinilla, 2007). Al revisar los porcentajes de inhibición radicular (Figura 4C), se evidencian resultados similares con otros estudios que indican una alta toxicidad de la muestra (Rodríguez Moreno et al., 2014). El desarrollo del hipocótilo arroja un porcentaje de inhibición negativo que evidencia que la longitud resulta 
ser más alta que en condiciones normales (control positivo) (Figura 4B); esto se puede deber a un proceso de hormesis por el que pudo atravesar la semilla y no debe interpretarse como algo beneficioso o estimulante, ya que ciertos compuestos presentes en la contaminación hídrica a bajas concentraciones producen exaltación por micronutrientes como cobre y zinc (Castillo Morales, 2004).
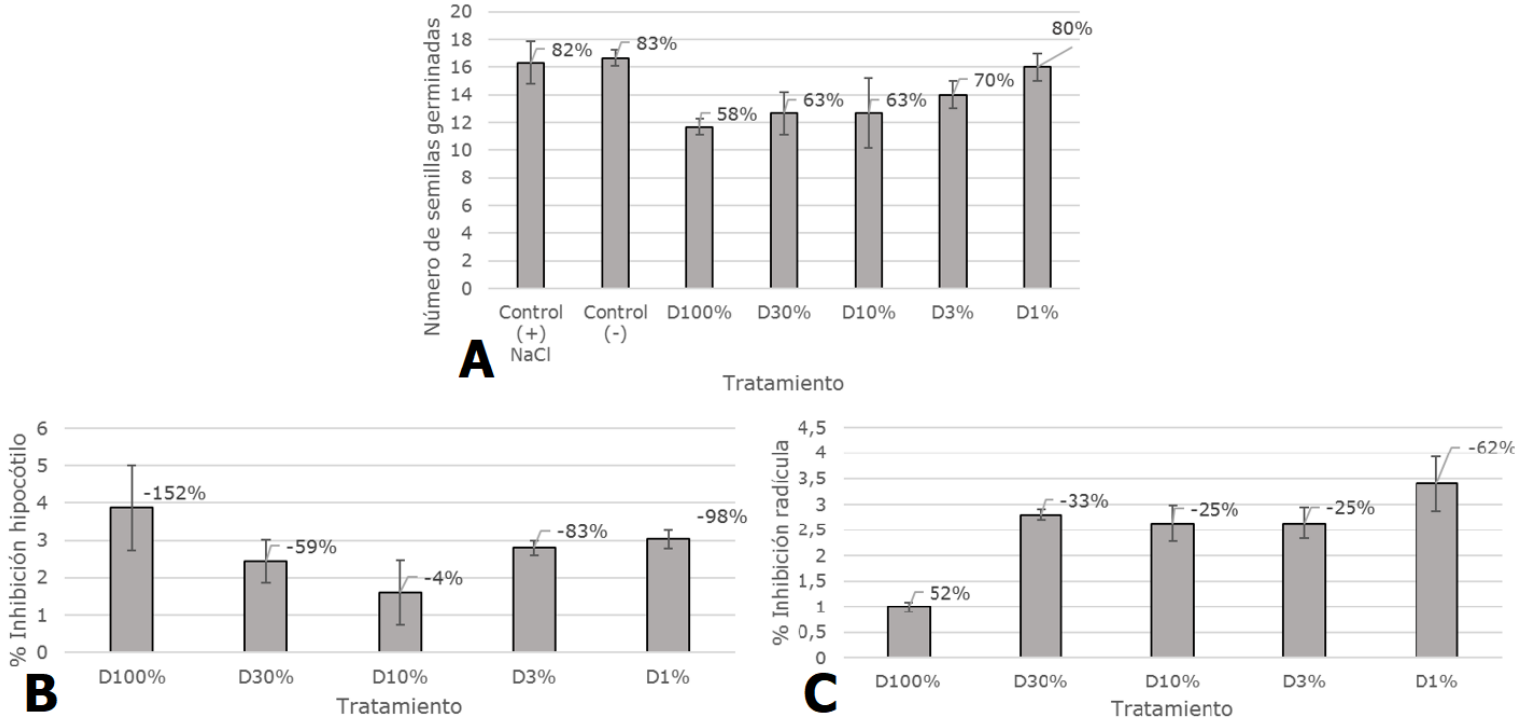

Figura 4. Porcentaje de inhibición de germinación (A), del hipocólito (B) y radicular (C).

Este estudio brinda una línea base, desde un experimento casero, para realizar trabajos de mayor profundidad muestral y estadística, así como la complementariedad con estudios de composición química y postulación de bioindicadores que permitan elucidar con mayor facilidad el impacto por metales pesados, que es el grupo de contaminantes más probable (Alcaldía local Puente Aranda, 2012; Pachón y Sarmiento Vela, 2008), y relacionarlos con los estudios de toxicología del lugar. La detección de metales a través de espectroscopia de adsorción atómica y fluorescencia atómica, espectrometría acoplado a masas o biosensores electroquímicos son métodos de alta precisión que permitirían elucidar con mayor precisión la presencia de los metales pesados, entre otros, posibles contaminantes en el agua (Gumpu et al., 2015). Por otro lado, 
algunos organismos son bioindicadores importantes de contaminación de metales pesados, como los peces y los briófitos, pues acumulan estos compuestos en sus cuerpos y por ende su abundancia y salud soportan el impacto negativo de la contaminación del agua (Authman et al., 2015; Zapata Muñoz et al., 2018).

\section{Referencias bibliográficas}

Authman, M. M. N., Zaki, M. S., Khallaf, E. A., y Abbas, H. H. (2015) Use of Fish as Bio-indicator of the Effects of Heavy Metals Pollution. J Aquac Res Development, 6(4), 328. doi: 10.4172/2155-9546.1000328. Recuperado de https://www.omicsonline.org/open-access/use-of-fish-asbioindicator-of-the-effects-of-heavy-metals-pollution-2155-95461000328. php?aid $=47054$

AccuWeather. (2018). [El tiempo en Colombia]. Recuperado de https://www.accuweather.com/es/co/colombia-weather

Alcaldía local Puente Aranda. (2012). PLAN AMBIENTAL LOCAL PUENTE ARANDA. Recuperado de http://ambientebogota.gov.co/documents/10157/2883174/PAL+P uente+Aranda+2013-2016.pdf

Beltrán Pineda, M.E., y Gómez Rodríguez, A.M (2014). Metales pesados ( $\mathrm{Cd}, \mathrm{Cr}$ y $\mathrm{Hg}$ ): su impacto en el ambiente y posibles estrategias biotecnológicas para su remediación. Revista I3+, 2(2), 82-112. doi: $10.24267 / 23462329.113$. Recuperado de http://revistasdigitales.uniboyaca.edu.co/index.php/reiv3/article/v iew/113

Bohórquez Echeverry, P., y Campos Pinilla, C. (2007). Evaluación de Lactuca sativa y Selenastrum capricornutum como indicadores de toxicidad en aguas. Universitas Scientiarum, 12(2), 83-98.

Recuperado de http://revistas.javeriana.edu.co/index.php/scientarium/article/vie w/4868

Castillo Morales, G. (2004). Ensayos toxicológicos y métodos de evaluación de calidad de aguas: estandarización, intercalibración, resultados y aplicaciones. Ottawa: IDRC. Recuperado de https://www.idrc.ca/sites/default/files/openebooks/1477/index.html

Google. (s.f.). [Mapa de Parque Milenta, Bogotá, Colombia en Google maps]. Recuperado de 
https://www.google.com/maps/place/Parque+Milenta/@4.616721 2,-

$74.1248954,434 \mathrm{~m} / \mathrm{data}=! 3 \mathrm{~m} 1 ! 1 \mathrm{e} 3 ! 4 \mathrm{~m} 5 ! 3 \mathrm{~m} 4 ! 1 \mathrm{~s} 0 \times 8 \mathrm{e} 3 \mathrm{f9eb} 3455 \mathrm{c} 5$ 1f3:0xaabce93c2a3648b1!8m2!3d4.6136627!4d-74.1247619

Gumpu, M. B., Sethuraman, S., Krishnan, U. M., Balaguru Rayappan, J.

B. (2015). A review on detection of heavy metal ions in water - An electrochemical approach. Sensors and Actuators B: Chemical, 213, 515-533. doi: 10.1016/j.snb.2015.02.122. Recuperado de https://www.sciencedirect.com/science/article/pii/S092540051500 307X

Guzmán Lenis, A. (2016). Toxicología ambiental. Conceptos clave para el desarrollo del curso. [OVI]. Recuperado de http://hdl.handle.net/10596/10487

IDEAM. (2002). Guía para el monitoreo de vertimientos, aguas superficiales y subterráneas. Recuperado de http://www.corponor.gov.co/control calidad/2014/Guia monitore o IDEAM.pdf

Moreno Rojas, S., Nieto Escalante, J. A., Pizano Callejas, J. E., Velandia Sepúlveda, O., Reyes Ávila, O. A., Bello Espinosa, A., y otros. (2008). Calidad del sistema hídrico de Bogotá. Bogotá: Editorial Universidad Javeriana: Alcaldía Mayor de Bogotá, Secretaria Distrital de Ambiente: Empresa de Acueducto y Alcantarillado de Bogotá. Recuperado de http://ambientebogota.gov.co/documents/24732/3987336/Calidad + del+sistema+hidrico+de+Bogot\%C3\%A1.pdf

Pachón, J.E., y Sarmiento Vela, H. (2008). Análisis espacio-temporal de la concentración de metales pesados en la localidad de Puente Aranda de Bogotá-Colombia. Revista Facultad de Ingeniería, 43, 120-133. Recuperado de

http://aprendeenlinea.udea.edu.co/revistas/index.php/ingenieria/a rticle/view/18764

Reyes, Y. C., Vergara, I., Torres, O. E., Díaz-Lagos, M., y González, E. E. (2016). Contaminación por metales pesados: Implicaciones en salud, ambiente y seguridad alimentaria. Revista Ingeniería Investigación y Desarrollo, 16(2), 66-77. Recuperado de https://revistas.uptc.edu.co/index.php/ingenieria_sogamoso/articl e/view/5447

Rodríguez Romero, A. J., Robles Salazar, C. A., Ruíz Picos, R. A., Lopez Lopez, E., Sedeño Díaz, J. E., y Rodriguez Dorantes, A. (2014). Índices de germinación y elongación radical de Lactuca sativa en el biomonitoreo de la calidad del agua del río Chalma. Revista 
internacional de contaminación ambiental, 30(3), 307-316.

Recuperado de

http://www.scielo.org.mx/scielo.php?script=sci arttext\&pid=S018

8-49992014000300007

SDP. (s.f.). TOMO I Diseño de la estrategia de intervención integral y multidimensional en materia socioeconómica, ambiental y urbanística para el río Fucha y su área de entorno. Recuperado de http://www.sdp.gov.co/sites/default/files/tomo1 final.pdf

Sobrero, M. C., \& Ronco, A. (2004). Ensayo de toxicidad aguda con semillas de lechuga (Lactuca sativa L.). En: Castillo, G. Ensayos toxicológicos y métodos de evaluación de calidad de aguas.

Canadá: IDRC, IMTA 71-79. Recuperado de http://www2.inecc.gob.mx/publicaciones2/libros/573/cap4.pdf

Tovar Artunduaga, M., Losada Salazar, G. M., \& García, T. F. (2015). Impacto en la salud por el inadecuado manejo de los residuos peligrosos. Ing. USBMed, 6(2), 46-50. Recuperado de http://www.revistas.usb.edu.co/index.php/IngUSBmed/article/vie w/1731/1497

Zapata Muñoz, Y. L., Trrujillo González, J. M., y Torres Mora, M. A. (2018). Distribución espacial del plomo (Pb) en el municipio de Villavicencio usando briófitos como medio de verificación de la calidad ambiental urbana. Revista de Investigación Agraria y Ambiental, 9(2), 269-280. doi: 10.22490/21456453.2167. Recuperado de http://hemeroteca.unad.edu.co/index.php/riaa/article/view/2167 